\title{
Stomach Mass
}

National Cancer Institute

\section{Source}

National Cancer Institute. Stomach Mass. NCI Thesaurus. Code C35491.

An abnormal growth located in the stomach. 